\title{
Polyelectrolyte Behavior of Poly(vinyl alcohol) in Aqueous Borax Solutions
}

\author{
Hiroshi OCHIAI, ${ }^{*}$ Yoshifumi FUJINO, ${ }^{*}$ Yuji TADOKORO, ${ }^{* *}$ \\ and Ichiro MURAKAMI* \\ *Department of Chemistry, Faculty of Science, Hiroshima University, \\ Higashisenda-machi, Naka-ku, Hiroshima 730, Japan. \\ ${ }^{* *}$ Chemistry Laboratory, Faculty of Integrated Arts and Sciences, Hiroshima University, \\ Higashisenda-machi, Naka-ku, Hiroshima 730, Japan.
}

(Received June 9, 1981)

\begin{abstract}
KEY WORDS Poly(vinyl alcohol) / Borate Ion / Polyelectrolyte Behavior / Limited Expansion /
\end{abstract}

Interactions between poly(vinyl alcohol) (PVA) and borate ions in aqueous media have been studied by many authors to elucidate the mechanisms of thickening and gelling of PVA solution caused by the addition of boric acid or its sodium salt (borax). Comprehensive reviews of the studies on the reaction between borate ion $\left(\mathrm{B}(\mathrm{OH})_{4}{ }^{-}\right)$and polyols such as glycols or sugars generally show that PVA interacts with borate ions to form two types of complexes, monodiol and didiol. ${ }^{1,2}$ Both complexes, however, have relatively low formation constants, and the constant for monodiol is considerably low in comparison with that for didiol. ${ }^{3}$ When such complexes are formed, some distribution of electrostatic charges may be expected to take place on the PVA chain as a result of negatively charged borate ions incorporated into the chain. Polyelectrolyte behavior, therefore, should be observed in such PVA solutions, though PVA itself is a typical neutral polymer. At the same time, complexation probably induces a viscosity decrease or precipitation of polymer as was observed in the systems of PVA and divalent metal ions., ${ }^{4,5}$ However, most of the viscometric studies on the PVA-borax systems have directed little attention to the expected polyelectrolyte behavior not explicitly manifest in the absence of a second added salt. ${ }^{6-8}$ In fact, in our previous viscometric study for the systems of various mole ratios of borax to PVA without another added salt, no explicit polyelec- trolyte behavior was observed except for an increase in the intrinsic viscosity with the mole ratio, and the results were interpreted rather ambiguously as a gain in excluded volume by interaction between PVA and borate ions. ${ }^{7}$

In the present study, the viscosity behavior of PVA in a borax solution has been investigated in both the presence and absence of sodium chloride $(\mathrm{NaCl})$. The observed viscosity behavior was interpreted in terms of a limited chain-expansion due to charges and intramolecular crosslinking simultaneously introduced in the PVA chain when the chain complexed with negatively charged borate ions, and an added-salt effect of free borax.

\section{EXPERIMENTAL}

PVA ( $99.9 \mathrm{~mol} \%$ saponified) was crudely fractionated with $n$-propanol-water systems into three fractions, which were purified by dialysis. The middle fraction was chosen; its viscosity average molecular weight was $1.23 \times 10^{5} .^{9}$ Anhydrous sodium tetraborate, $\mathrm{Na}_{2} \mathrm{~B}_{4} \mathrm{O}_{7}$, (borax) and sodium chloride $(\mathrm{NaCl})$ were used after heat-treatment. Since PVA is not easily soluble in cold water, it was dissolved in water at an elevated temperature to prepare a dilute stock solution; no gelation developed even in the presence of excess borax. The usual dilution procedure was applied to keep the borax concentration $c_{\mathrm{b}}$ and/or $\mathrm{NaCl}$ concentration $c_{\mathrm{s}}$ (in $\mathrm{moll}^{-1}$ ) con- 


\section{H. OCHIAI et al.}

stant throughout a series of viscosity measurements.

Viscosity measurements were made on a Ubbelohde-type capillary viscometer with a suspended level (the flow time for water was $255.2 \mathrm{~s}$ at $25^{\circ} \mathrm{C}$ ). The kinetic energy correction was negligible. The measurement temperature was maintained at $(25 \pm 0.02)^{\circ} \mathrm{C}$.

\section{RESULTS AND DISCUSSION}

Figure 1 shows the dependence of reduced viscosity $\eta_{\mathrm{sp}} / c_{\mathrm{p}}$ on polymer concentration $c_{\mathrm{p}}$ at various concentrations of borax $c_{\mathrm{b}}$. The reduced viscosity increased with an increase in $c_{\mathrm{b}}$, suggesting the existence of interactions between PVA and borax, i.e., complexation of $\mathrm{OH}$ groups of PVA with borate ions. Since the present $c_{\mathrm{p}}$ was not high enough to cause intermolecular crosslinking by interactions with borate ions, the observed increase in $[\eta]$ may be ascribed to intramolecular interactions between the PVA chain and borate ions. In fact, the concentration distributions at sedimentation equilibrium gave the same molecular weight for PVA in solutions dialyzed with $0.06 \mathrm{moll}^{-1}$ borax and without borax, and exhibited no evidence of aggregation by intermolecular crosslinks. ${ }^{10}$ If the

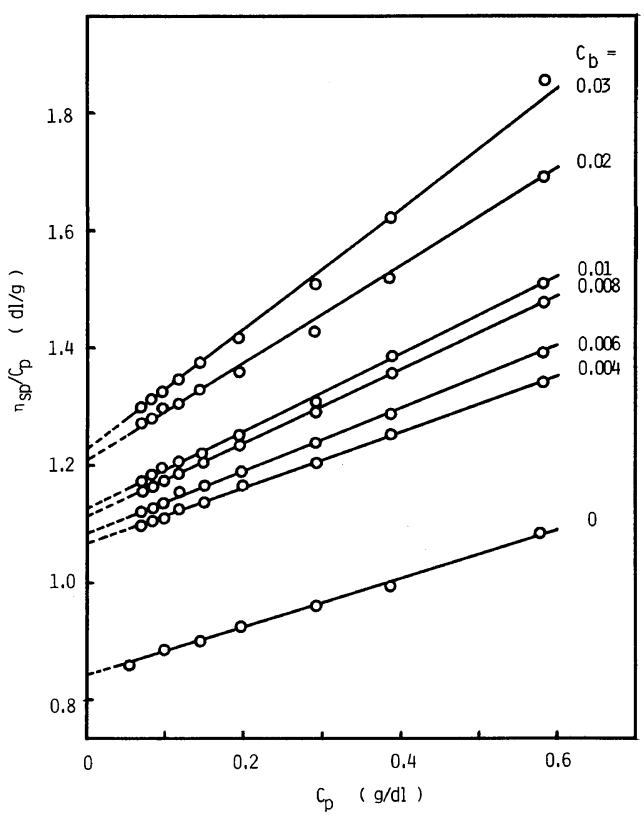

Figure 1. Typical viscosity plots for PVA solutions with various borax concentrations $c_{\mathrm{b}}$ (in $\mathrm{mol}^{-1}$ ).
PVA chain is endowed with a polyelectrolyte character through complexation with negatively charged borate ions, polyelectrolyte behavior, such as an upswing in the viscosity plot at low polymer concentrations, will be observed. However, what we observed in this study was that the viscosity plots were linear down to the lowest $c_{\mathrm{p}}$ examined. A similar result was also obtained in the previous study. ${ }^{7}$

When $\mathrm{NaCl}$ was added in addition to borax, the viscosity increase due to the presence of borax was remarkably suppressed, and the degree of which was greater for higher $\mathrm{NaCl}$ concentration $c_{\mathrm{s}}$. A typical example is shown in Figure 2. The data are for $c_{\mathrm{b}}=0.02 \mathrm{moll}^{-1}$, where the viscosity plots exhibit straight lines with similar slopes. At higher $c_{\mathrm{s}},[\eta]$ is smaller than that of PVA in the absence of both borax and $\mathrm{NaCl}$. Phase separation (not gelation) was observed at still higher $c_{\mathrm{s}}$. A similar change in $[\eta]$ with the addition of $\mathrm{NaCl}$ was observed at other $c_{\mathrm{b}}\left(=0.005\right.$ and $\left.0.01 \mathrm{moll}^{-1}\right)$. The critical $c_{\mathrm{s}}$ for the onset of phase separation was found to be $0.15-0.4 \mathrm{moll}^{-1} \mathrm{NaCl}$, and was higher for higher $c_{\mathrm{b}}$ in the range studied. However, unless

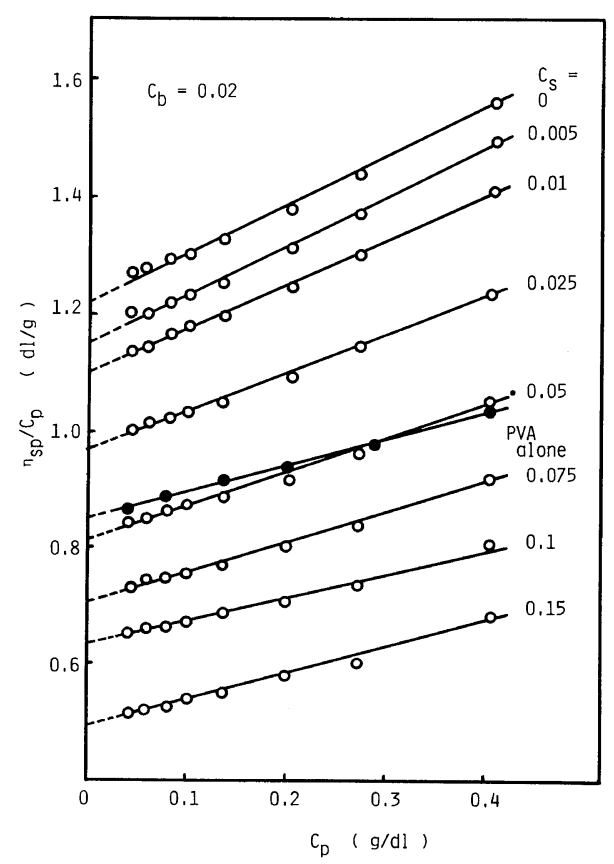

Figure 2. Typical viscosity plots for PVA solutions of $c_{\mathrm{b}}=0.02$ at various $\mathrm{NaCl}$ concentrations $c_{\mathrm{s}}$ (in $\mathrm{moll}^{-1}$ ). Filled circles show pure PVA $\left(c_{\mathrm{b}}, c_{\mathrm{s}}=0\right)$. 


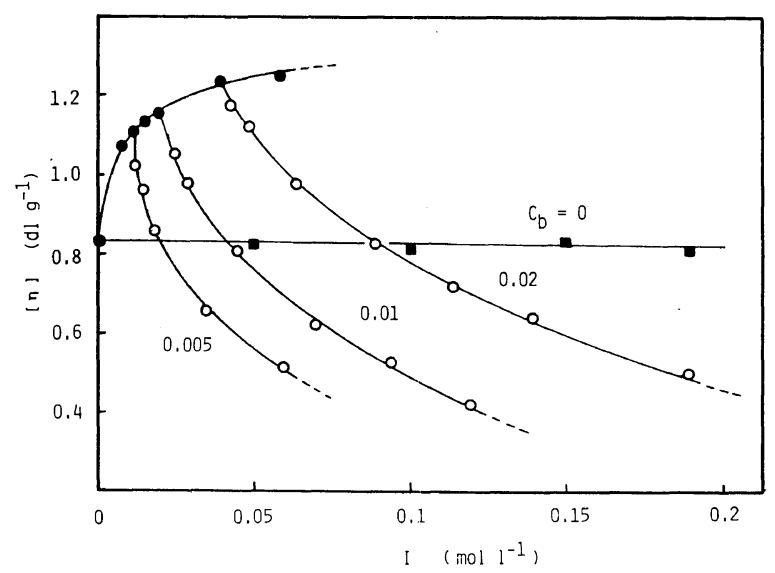

Figure 3. Dependence of intrinsic viscosity of PVA on ionic strength $I$ in solutions with various borax and $\mathrm{NaCl}$ concentrations: $\mathrm{O}, \mathrm{PVA}-$ borax- $\mathrm{NaCl}$;, PVA-borax; $\mathbf{Q}$, PVA-NaCl.

borax was present, no phase separation occurred in the range of $c_{\mathrm{s}}$ examined, and $[\eta]$ remained constant within experimental error. Thus, it seems that the effect of $\mathrm{NaCl}$ added to aqueous PVA solution manifests itself only in the presence of borax. The observed changes in $[\eta]$ and the phase separation are quite similar to those usually observable when a simple salt is added to a polyelectrolyte solution.

To visualize the dependence of $[\eta]$ on $c_{\mathrm{b}}$ and/or $c_{\mathrm{s}}$, the values of $[\eta]$ are plotted against ionic strength $I$ in Figure 3. In calculating $I$, the concentrations of both $\mathrm{Na}^{+}$and $\mathrm{B}(\mathrm{OH})_{4}{ }^{-}$in a borax solution were taken equal to $2 c_{\mathrm{b}}$, since the chemical constitution of a dilute solution of borax $\left(\mathrm{Na}_{2} \mathrm{~B}_{4} \mathrm{O}_{7}\right)$ is the same as that of boric acid half-neutralized with sodium hydroxide, ${ }^{11,12}$ and since the binding of borate ion to PVA in a dilute solution is not very high. ${ }^{3,13}$ In systems containing only borax, $[\eta]$ increases sharply in the range of lower I, i.e., lower $c_{\mathrm{b}}$, but at higher $c_{\mathrm{b}}$ this increase becomes so slight as to appear leveled off. This increasing trend of $[\eta]$ with $c_{b}$ is quite similar to the binding isotherms of borate ion to PVA as shown previously from an equilibrium dialysis study, ${ }^{13}$ in which the binding was almost saturated at about $0.05 \mathrm{moll}^{-1}$ borax. Thus, the observed increase in $[\eta]$ can be ascribed to the complexation of borate ions with PVA. On the contrary, in systems containing both borax and $\mathrm{NaCl},[\eta]$ shows a sharp decrease in the range of lower $I$, and this decrease in $[\eta]$ is more pronounced as $c_{\mathrm{b}}$ is lowered. At higher $I$ (higher $c_{\mathrm{s}}$ ), $[\eta]$ for all systems becomes smaller than $0.843 \mathrm{dlg}^{-1}$ for the present PVA in pure water. This fact suggests that the PVA chain is contracted to an unusual extent under such conditions. Extrapolation of $[\eta]$ to infinite ionic strength on the $[\eta] v s .1 / I$ plot gives $[\eta]_{\infty}$ of $0.2-0.3 \mathrm{dlg}^{-1}$ (the usual $[\eta] v s .1 / I^{1 / 2}$ plot gave unreasonable negative intercepts). The values of $[\eta]_{\infty}$ so obtained for PVA in borax solutions are very small in comparison with the unperturbed value in pure water, i.e., $[\eta]_{\theta}=0.56 \mathrm{dlg}^{-1}$, which may be expected from $\mathrm{K}_{0}=1.6 \times 10^{-3} \mathrm{dl} \mathrm{g}^{-1}$ estimated by the Stockmayer-Fixman extrapolation ${ }^{9,14}$ and the molecular weight of $1.23 \times 10^{5}$ for our PVA sample.

The above-mentioned viscosity changes of aqueous PVA solution with the addition of borax and/or $\mathrm{NaCl}$ lead us to conclude that the present systems behave as polyelectrolyte solutions. However, PVA in the present systems should not be treated as a simple linear polyelectrolyte whose chain conformation is affected by charge repulsion resulting from the incorporation of borate ions and also by charge shielding due to coexisting free borax and added $\mathrm{NaCl}$. In addition to these charge effects, changes in chain flexibility caused by the PVAborax complexations should also be taken into consideration, since borate ions tend to complex favorably with the $\mathrm{OH}$ groups of 1,2- or 1,3structures owing to its tetrahedral structure. ${ }^{2,16}$ Unfortunately, there is as yet no theory applicable to such a macroion system. ${ }^{15}$

Complexation of the monodiol type, which may occur mainly at adjacent $\mathrm{OH}$ groups along the PVA chain, would hinder the rotation about the skeletal 


\section{H. OCHIAI et al.}

bonds in complexed moieties. This may have a rather expansive effect on the chain. On the other hand, complexation of the didiol type would have a contractive effect on the chain, since this complexation should occur at any two pairs of adjacent $\mathrm{OH}$ groups to introduce some intramolecular crosslinks in the chain (rather long-range) ${ }^{2,16}$ The increase in $c_{\mathrm{b}}$ may increase not only the charge density on the PVA chain but also the crosslinking density. Therefore, chain expansion due to charge repulsion and/or the complexation of the monodiol type may be limited at such crosslinking points. Thus, the observed viscosity increase and its leveling-off with $c_{\mathrm{b}}$ may be attributed to this limited expansion of the PVA chain complexed with negatively charged borate ions. The addition of $\mathrm{NaCl}$ to PVA-borax systems shields away the charge effect and allows the PVA chain to contract, leaving the crosslinking effect. At higher $I$, where the expansive contribution from the monodiol complex may be minor, only the crosslinking effect remains, leading to an unusually small coil size. Moreover, it should be noted that the free borax itself may prevent the chain from electrostatic expansion, especially in systems without $\mathrm{NaCl}$, even if $c_{\mathrm{b}}$ is not very high and $I$ is correspondingly low. This effect of free borax and the limited expansion by crosslinking may be a possible explanation as to why no polyelectrolyte character can be observed in the viscosity plots shown in Figure 1.

Thus, the thickening of aqueous PVA by borax may be interpreted qualitatively to some extent as the complex effects of added borax. The electrostatic expansion due to charges on the polymer chain and the limited expansion due to the intramolecular crosslinks come about simultaneously. by complexation with borate ions, and free borax acts as a shield against repulsion between charges on the chain.

\section{REFERENCES}

1. H. Deuel and H. Neukom, Makromol. Chem., 3, 13 (1949).

2. C. A. Finch, "Polyvinyl Alcohol: Properties and Applications," Wiley, London, 1973, Chapter 9.

3. H. Ochiai, S. Shimizu, Y. Tadokoro, and I. Murakami, Polymer, 22, 1456 (1981).

4. H. Shirai and N. Hojo, J. Chem. Soc. Jpn., 1972, 1954.

5. N. Hojo, H. Shirai, and S. Hayashi, J. Polym. Sci., Polym. Symp., No. 47, 299 (1974).

6. K. Bolewski and B. Rychly, Kolloid Z. Z. Polym., 288, 48 (1968).

7. I. Murakami, Y. Fujino, H. Ochiai, and Y. Tadokoro, J. Polym. Sci., Polym. Phys. Ed., 18, 2149 (1980).

8. R. F. Nickerson, J. Appl. Polym. Sci., 15, 111 (1971).

9. B. J. R. Scholtens and B. Bijsterbosch, J. Polym. Sci., Polym. Phys. Ed., 17, 1771 (1979).

10. H. Ochiai, Y. Fujino, Y. Tadokoro, and I. Murakami, Polym. Prepr., Jpn., 27, 987 (1978).

11. G. L. Roy, A. L. Laferriere, and J. O. Edwards, J. Inorg. Nucl. Chem., 4, 106 (1957).

12. J. M. Conner and V. C. Bulgrin, J. Inorg. Nucl. Chem., 29, 1953 (1967).

13. H. Ochiai, Y. Fujino, Y. Tadokoro, and I. Murakami, Polymer, 21, 485 (1980).

14. L. K. Koopal, Colloid Polym. Sci., 259, 490 (1981).

15. For examples, S. A. Rice and M. Nagasawa, "Polyelectrolyte Solutions," Academic Press, London and New York, 1961; F. Oosawa, "Polyelectrolytes," Marcel Dekker, New York, 1971.

16. H. S. Isbell, J. F. Brewster, N. B. Holt, and H. L. Frush, J. Res. Natl. Bur. Stand., 40, 129 (1948). 